\title{
Association of Traditional Cardiovascular Risk Factors with Venous Thromboembolism: An Individual Participant Data Meta- analysis of Prospective Studies
}

\author{
Bakhtawar K. Mahmoodi, MD PhD MPH ${ }^{1,2}$, Mary Cushman, MD MSc ${ }^{3}$, Inger Anne Næss, MD \\ $\mathrm{PhD}^{4}$, Matthew A. Allison, MD MPH${ }^{5}$, Willem Jan Bos, MD PhD ${ }^{1}$, Sigrid K. Brækkan, $\mathrm{PhD}^{6}$, \\ Suzanne C. Cannegieter, MD PhD7, Ron T. Gansevoort, MD PhD ${ }^{8}$, Philimon N. Gona, PhD \\ $\mathrm{MPH}^{9}$, Jens Hammerstrøm, MD PhD ${ }^{4}$, John-Bjarne Hansen, MD $\mathrm{PhD}^{6}$, Susan Heckbert, MD \\ PhD ${ }^{10}$, Anders G. Holst, MD PhD ${ }^{11}$, Susan G. Lakoski, MD MS ${ }^{12}$, Pamela L. Lutsey, PhD ${ }^{13}$, \\ JoAnn E. Manson, MD DrPH ${ }^{14}$, Lisa W. Martin, MD ${ }^{15}$, Kunihiro Matsushita, MD PhD ${ }^{16}$, \\ Karina Meijer, MD PhD ${ }^{2}$, Kim Overvad, MD PhD ${ }^{17}$, Eva Prescott, MD DMSc ${ }^{18}$, Marja \\ Puurunen, MD PhD ${ }^{19}$, Jacques E. Rossouw, MD ${ }^{20}$, Yingying Sang, MSc ${ }^{16}$, Marianne T. \\ Severinsen, MD PhD ${ }^{21}$, Jur ten Berg, MD PhD ${ }^{1}$, Aaron R. Folsom, MD MPH ${ }^{13}$, and Neil A. \\ Zakai, MD MSc ${ }^{3}$
}

${ }^{1}$ Department of Cardiology and Internal Medicine, Sint Antonius Hospital, Nieuwegein, the Netherlands ${ }^{2}$ Department of Haematology, University Medical Center Groningen, University of Groningen, Groningen, the Netherlands ${ }^{3}$ Departments of Medicine and Pathology, University of Vermont, Burlington, Vermont, USA ${ }^{4}$ Departments of Hematology, Trondheim University Hospital, Trondheim, Norway ${ }^{5}$ Department of Family and Preventive Medicine, University of California San Diego, La Jolla, California, USA ${ }^{6}$ K.G.Jebsen - Thrombosis Research and Expertise Center (TREC) Department of Clinical Medicine, University of Tromsø, Tromsø, Norway ${ }^{7}$ Department of Clinical Epidemiology, Leiden University Medical Center, University of Leiden, Leiden, the Netherlands ${ }^{8}$ Department of Internal Medicine, University Medical Center Groningen, University of Groningen, Groningen, the Netherlands ${ }^{9}$ Department of Exercise and Health Sciences, University of Massachusetts, Boston, Massachusetts, USA ${ }^{10}$ Department of Epidemiology and Cardiovascular Health Research Unit, University of Washington, Seattle, Washington, USA ${ }^{11}$ Laboratory for Molecular Cardiology, Copenhagen University Hospital, Rigshospitalet, Copenhagen, Denmark ${ }^{12}$ Department of Clinical Cancer Prevention and Cardiology, the University of Texas MD Anderson Cancer Center, Houston, Texas, USA ${ }^{13}$ Division of Epidemiology and Community Health, University of Minnesota, Minneapolis, Minnesota, USA ${ }^{14}$ Department of Medicine, Brigham and Women's Hospital, Harvard Medical School, Boston,

\footnotetext{
Address for Correspondence: Bakhtawar K. Mahmoodi, Department of Cardiology, Sint Antonius Hospital, Koekoekslaan 1, 3435 CM Nieuwegein, The Netherlands, Phone 0031-611828388, Fax 0031-503611790, b.k.mahmoodi@umcg.nl.

Authors' contributions: BKM, MC and NZ conceived the study concept and design. BKM developed the statistical code, which was shared with the collaborating studies. Analyses of individual studies were performed by the representing co-author using the same centrally developed code. BKM meta-analyzed the estimates. All authors took part in the interpretation of the data. BKM, MC, and NZ, drafted the manuscript, and all authors provided critical revisions of the manuscript for important intellectual content.

Conflict of Interest Disclosures: None declared. All authors submitted the ICMJE form on Disclosure of Potential Conflicts of Interest.

The views expressed in this manuscript are those of the authors and do not necessarily represent the views of the National Heart, Lung, and Blood Institute; the National Institutes of Health; or the U.S. Department of Health and Human Services.
} 
Massachusetts, USA ${ }^{15}$ Division of Cardiology, George Washington University School of Medicine and Health Sciences, Washington DC, USA ${ }^{16}$ Johns Hopkins Bloomberg School of Public Health, Baltimore, Maryland, USA ${ }^{17}$ Department of Public Health, Section for Epidemiology, Aarhus University, Aarhus, and Department of Cardiology, Aalborg University Hospital, Aalborg, Denmark ${ }^{18}$ Department of Cardiology, Bispebjerg Hospital, University of Copenhagen, Copenhagen, Denmark ${ }^{19}$ Framingham Heart Study of Boston University School of Medicine and NHLBI, Framingham, Massachusetts, USA ${ }^{20}$ National Heart, Lung, and Blood Institute, Bethesda, Maryland, USA ${ }^{21}$ Department of Hematology, Aalborg University Hospital, Aalborg, Denmark

\section{Abstract}

Background-There is much controversy surrounding the association of traditional cardiovascular disease (CVD) risk factors with venous thromboembolism (VTE).

Methods-We performed an individual level random-effect meta-analysis including 9 prospective studies with measured baseline CVD risk factors and validated VTE events. Definitions were harmonized across studies. Traditional CVD risk factors were modeled categorically, as well as continuously using restricted cubic splines. Estimates were obtained for overall VTE, provoked (i.e., VTE occurring in the presence of one or more established VTE risk factors) and unprovoked VTE, pulmonary embolism (PE) and deep-vein thrombosis (DVT).

Results-The studies included 244,865 participants with 4,910 VTE events occurring during a mean follow-up 4.7-19.7 years per study. Age, sex, and body-mass index adjusted hazard ratios for overall VTE were 0.98 (95\%CI, 0.89-1.07) for hypertension, 0.97 (0.88-1.08) for hyperlipidemia, $1.01(0.89-1.15)$ for diabetes and $1.19(1.08-1.32)$ for current smoking. After full adjustment these estimates were numerically similar. When modeled continuously, an inverse association was observed for systolic blood pressure (HR=0.79 [95\% CI, 0.68-0.92] at systolic blood pressure 160 vs. $110 \mathrm{mmHg}$ ), but not for diastolic blood pressure or lipid measures with VTE. An important finding from VTE subtype analyses was that cigarette smoking was associated with provoked but not with unprovoked VTE. Fully adjusted hazard ratios for the associations of current smoking with provoked and unprovoked VTE were 1.36 (95\% CI, 1.22-1.52) and 1.08 (0.90-1.29), respectively.

Conclusions-Except the association of cigarette smoking with provoked VTE, which is potentially mediated through comorbid conditions such as cancer, the modifiable traditional CVD risk factors are not associated with increased VTE risk. Higher systolic blood pressure showed inverse association with VTE.

\section{Keywords}

Venous Thromboembolism; Thrombosis; Cardiovascular disease; Risk factors; Hypertension; Diabetes; Hyperlipidemia; Smoking

\section{Introduction}

Each year, over 500,000 individuals in the United States and European Union die from venous thromboembolism (VTE). ${ }^{1,2}$ Among VTE survivors 50\% have long-term 
complications. ${ }^{1,2}$ VTE, consisting of deep vein thrombosis (DVT) or pulmonary embolism $(\mathrm{PE})$, is clinically defined as either provoked or unprovoked. Provoked events are preceded by triggering, generally transient, risk factors such as immobilization, surgery, major trauma, or cancer. ${ }^{3}$ About $50 \%$ of VTE occur in the absence of any risk factors and are classified as unprovoked. ${ }^{4}$ Apart from the aforementioned provoking factors, older age, family history of VTE, certain genetic variants, oral contraceptive use and obesity are also known major VTE risk factors.

In contrast, arterial thromboembolism, comprising coronary heart disease, stroke, and peripheral artery disease mainly occurs with atherosclerosis, which is primarily driven by the major modifiable traditional cardiovascular disease (CVD) risk factors, including hypertension, hyperlipidemia, diabetes, and smoking. ${ }^{5}$ The traditional CVD risk factors and VTE share some common lifestyle risk factors such as physical inactivity and obesity. Nevertheless, VTE and CVD have historically been viewed as two different diseases with distinct risk factors. ${ }^{6}$

In the last decade, several studies on the associations of CVD risk factors with VTE risk have been conducted with inconclusive results. ${ }^{6-16}$ In 2008, a meta-analysis showed positive associations for hypertension, diabetes, hyperlipidemia and smoking with VTE incidence. ${ }^{8}$ However, this meta-analysis did not adjust for important confounders such as age and body mass index and included primarily studies with case-control design and non-validated outcomes. To obtain robust evidence with minimal bias, we performed an individual-level data meta-analysis of prospective studies in which traditional CVD risk factors were measured and VTE events validated.

\section{Methods}

\section{Study selection criteria}

Eligible studies had to be prospective cohorts or clinical trials with measured CVD risk factors and validated VTE events. A PubMed search was performed on October 21, 2014 with terms for each traditional CVD risk factor and for VTE excluding newborns and infants (search strategy described on page 3 of the online Data Supplement). Results were restricted to English language, humans and publication date after January 1, 1980 (since reliable diagnostic modalities for VTE were not widely available before 1980). Of 3,192 publications (Figure 1) screened by reading the titles and abstracts, 46 studies were selected for full text review, with 11 meeting eligibility criteria. Another two unpublished cohort studies were identified via personal contacts. Of the 13 that met the inclusion criteria of being prospective studies with data on measured CVD risk factors and validated VTE events, two studies were unable to provide data, ${ }^{17,18}$ and two did not reply to our invitation. ${ }^{19,20}$ Therefore, 9 studies were included in the meta-analysis. ${ }^{13-16,21-25}$

\section{Outcome variables definitions}

Only objectively verified, symptomatic and validated VTE events were included. DVT was confirmed by duplex ultrasound or venography, and PE by ventilation/perfusion lung scanning, angiography, spiral computed tomography or autopsy. Patients with PE and 
concurrent DVT were included in the PE group. We defined provoked or unprovoked VTE based on each study's definition. Major trauma, surgery, significant immobilization or active cancer in the preceding 3 months were the main determinants for classifying VTE as provoked. Some cohorts included additional exposures to define provoked VTE, such as the use of oral contraceptives or hormone therapy, pregnancy, long-distance travel, active infectious disease, acute myocardial infarction, paresis/paralysis of the leg, and heart failure (see online-only Data Supplement pages 3-8). In each study, in the absence of study-defined provoking factors, VTE was classified as unprovoked.

\section{Exposure variables definitions}

Risk factor definitions were harmonized across studies. Hypertension was defined as systolic blood pressure $\geq 140 \mathrm{~mm} \mathrm{Hg}$, diastolic blood pressure $\geq 90 \mathrm{~mm} \mathrm{Hg}$, or use of antihypertensive medication. Diabetes mellitus was defined as fasting glucose concentration $\geq 7.0 \mathrm{mmol} / \mathrm{L}$ ( $\geq 126 \mathrm{mg} / \mathrm{dL}$ ), non-fasting glucose concentration $\geq 11.1 \mathrm{mmol} / \mathrm{L}$ ( $\geq 200 \mathrm{mg} / \mathrm{dL}$ ) or use of glucose lowering drugs or self-reported diabetes. Hyperlipidemia was defined as total cholesterol $\geq 5.0 \mathrm{mmol} / \mathrm{L}$ ( $\geq 193 \mathrm{mg} / \mathrm{dL}$ ) in patients with a history of CVD and as $\geq 6.0$ $\mathrm{mmol} / \mathrm{L}$ ( $2232 \mathrm{mg} / \mathrm{dL}$ ) in patients without history of CVD, or use of lipid-lowering medication. History of CVD was defined as previous myocardial infarction, coronary revascularization, stroke, or peripheral artery disease objectively verified by diagnostic modalities, revascularization or amputations due to ischemia. Smoking was dichotomized as self-reported current smoking versus former or never-smoking combined, and as former smoking versus never-smoking. Body-mass index (BMI) was calculated as body weight in kilograms divided by squared height in meters.

\section{Statistical analysis}

Analyses were performed in two stages. First, each study analyzed their data with a centrally developed statistical code. The study-specific estimates and contrasts were shared with the study coordinator (BKM) to perform the meta-analysis. Cox proportional hazards regression was used to estimate the hazard ratios (HRs) of overall VTE and VTE subtypes. We tested three models: 1) unadjusted, 2) age, sex and BMI (continuous) adjusted, and 3) fully adjusted. The fully adjusted model included age, sex, race, BMI (continuous), history of CVD, history of VTE, hypertension, diabetes, hypercholesterolemia, and current and former smoking. If one or more of the variables listed for the fully adjusted model were not ascertained in a study, that specific variable was dropped from the list of the fully adjusted model variables for that study. To adjust for trial-arms or multiple sub-cohorts (e.g., Framingham Heart Study), Cox models with strata option were fit, with the strata variable representing the randomization status or the sub-cohorts.

In addition to categorical analyses, systolic blood pressure, diastolic blood pressure, mean arterial pressure, pulse pressure, total cholesterol, low-density lipoprotein, high-density lipoprotein, and triglycerides were analyzed continuously using restricted cubic splines. The blood pressure models were adjusted for age, sex, BMI, history of CVD and antihypertensive drugs. Similarly, lipid measures were adjusted for age, sex, BMI, prior history of CVD, and use of lipid lowering medication. Knots and reference values for these variables were prespecified, and were partially based on the distribution of these variables in 
the REGARDS and PREVEND studies to avoid extreme knots outside the data-range. The same knots and reference values were used across all studies.

In sensitivity analysis, the associations of the exposure variables with the overall VTE and subtypes of VTE were assessed for the first five years of follow-up to assess whether the long follow-up available in most studies could have diluted the exposure-outcome risk associations.

In the second stage, the obtained estimates from individual studies were meta-analyzed using random-effect meta-analysis. If a study had zero events in a certain spline section, the estimate of that study for that particular spline section was dropped from the meta-analysis. Heterogeneity of the pooled estimates was assessed using the $\chi^{2}$ test for heterogeneity and the $I^{2}$ statistic. Potential sources of heterogeneity were explored by meta-regression analysis. In all analyses, a $P$ value of less than 0.05 was considered statistically significant. All analyses were conducted using Stata 12.2 (www.stata.com) and some figures were constructed with $\mathrm{R}$ version 2.14.1.

\section{Results}

Baseline characteristics of the 9 studies are shown in Table 1. Eight studies were community-based prospective cohort studies and one study consisted of two clinical trials including only women. Of the 244,865 participants in the analysis, a total of 4,910 developed VTE during the mean follow-up ranging from 4.7 to 19.7 years per study. Of 4,910 VTE events, $36 \%$ occurred within the first five years of follow-up. Overall, $44 \%$ of the VTE events were classified as unprovoked and $44 \%$ as PE with or without concurrent DVT (Table 1). Race was not considered as a covariate in the Framingham Heart Study and the European cohorts since $\geq 95 \%$ of participants in these studies were Caucasian. Unavailable data included prior history of CVD in one study, hyperlipidemia and lipid measures in one study and pre-baseline history of VTE in five studies. Whereas current smoking was available in all studies, in two studies former smoking status was not available. The proportion of missing values was $<1 \%$ for the majority of the variables (Online-only Data Supplement Table 1).

\section{Risk of VTE outcomes during the total follow-up}

Pooled estimates of associations of categorical CVD risk factors with VTE are shown in Figure 2. Except for current smoking, all variables showed clear positive associations with VTE in the unadjusted models. However, adjustment for age, sex and BMI resulted in elimination of VTE-risk associations for hypertension (HR=0.98 [95\% CI, 0.89-1.07]), hyperlipidemia (HR=0.97 [0.88-1.08]), diabetes $(H R=1.01$ [0.89-1.15]) and former smoking (HR= 0.99 [0.93-1.06]). Current smoking ( $H R=1.19$ [1.08-1.32]) was positively associated with overall VTE in this age, sex and BMI adjusted model. Estimates remained largely unchanged in fully adjusted models. Heterogeneity across studies tended to be moderate to high for the crude associations ( $I^{2}$ values ranging from $50 \%$ to $92 \%$ ), but low in the adjusted models, with an exception for current smoking ( $\left.I^{2}=51 \%\right)$. Using metaregression, of the study-level variables shown in Table 1, none of these explained the heterogeneity observed for current smoking in the fully adjusted overall VTE model. Results 
were generally similar for unprovoked versus provoked VTE, and PE versus DVT (Onlineonly Data Supplement Figures 1-4). Exceptions were that in the fully adjusted models current smoking was only associated with provoked (HR=1.36 [1.22-1.52], $\left.P^{2}=0 \%\right)$ and not with unprovoked (HR=1.08 [0.90-1.29], $\left.I^{2}=42 \%\right)$ VTE. Similarly, former smoking was only associated with provoked $\left(\mathrm{HR}=1.11[1.00-1.23], I^{2}=0 \%\right)$ but not unprovoked $(\mathrm{HR}=1.01$ $\left.[0.89-1.16], I^{2}=21 \%\right)$ VTE.

The associations of blood pressure and lipid measures were modeled continuously using restricted cubic splines (Figures 3 and 4). Whereas systolic and pulse pressure showed nearlinear inverse associations with VTE, diastolic and mean-arterial pressure showed inverse associations only at the lower ends of diastolic and mean-arterial pressure (Figure 3). Compared to the reference value of $110 \mathrm{mmHg}$, the hazard ratio for VTE was 0.79 (95\% CI, $0.68-0.92)$ at systolic blood pressure of $160 \mathrm{mmHg}$. The hazard ratio was $1.02(0.85-1.22)$ at diastolic blood pressure of $100 \mathrm{mmHg}$ as compared to the reference value of $75 \mathrm{mmHg}$. The inverse association of systolic blood pressure was somewhat more prominent for unprovoked as compared to provoked VTE (Online-only Data Supplement Figures 5 and 6) and for PE as compared to DVT (Online-only Data Supplement Figures 7 and 8). For lipid measures including total cholesterol, LDL, HDL and triglycerides, no clinically significant associations with overall VTE were observed (Figure 4). Also no major differences were observed for unprovoked versus provoked VTE or PE versus DVT (Online-only Data Supplement Figures 9-12). For glucose levels, there was a weak inverse relation in the normal glucose range but not at elevated glucose levels (Online-only Data Supplement Figure 13).

\section{Sensitivity analyses on the risk of VTE during the first 5-years of follow-up}

As compared to the total follow-up, the risk associations in the first 5-years of follow-up were generally comparable, except confidence intervals were slightly wider due to fewer events (Online-only Data Supplement Figures 14-29). The inverse association of systolic blood pressure during the first 5-years of follow-up was somewhat more prominent as compared to the total follow-up, especially for provoked VTE.

\section{Discussion}

This meta-analysis of 244,865 participants and 4,910 VTE events from 9 prospective studies demonstrated that, other than the association of smoking with provoked VTE, the modifiable traditional CVD risk factors (i.e., hypertension, hyperlipidemia, diabetes, and smoking) were not independently associated with overall or subtypes of VTE. While hypertensive status was not associated with VTE, higher systolic blood pressure was associated with a decreased risk of VTE, which was more obvious for unprovoked versus provoked VTE and for PE versus DVT. Continuously modeled lipid measures and glucose showed no meaningful associations with overall or subtypes of VTE.

Several studies have reported on the association of CVD risk factors with VTE. ${ }^{6-16}$ Due to inconsistent results, there is no agreement on whether traditional CVD risk factors are associated with incident VTE. This is the first meta-analysis of individual participant-level data of high quality prospective studies on this topic. All included cohort studies had the 
maximum score on the Newcastle - Ottawa Quality Assessment Scale of Cohort Studies.

Aggregated-level data meta-analyses have been published on the association of hypertension $(n=1)$, hyperlipidemia $(n=1)$, diabetes $(n=4)$, and smoking $(n=2)$ with VTE. ${ }^{8-12}$ Due to differences in definitions, study designs, covariates considered, inclusion of non-validated VTE events and inability to differentiate between VTE subtypes, the results of these metaanalyses are difficult to interpret. A meta-analysis by Ageno et al found positive associations with VTE for hypertension, hyperlipidemia, diabetes and smoking. ${ }^{8}$ However, this metaanalysis did not adjust for important confounders such as age and BMI, which were confirmed to have large impact on the results in our analyses. Meta-analyses on the associations of diabetes and smoking with VTE largely share the same limitations. ${ }^{9-12}$ Consistent with our findings, Bell et al. reported that the association of diabetes was not significant once the estimates were adjusted for age, sex and BMI. ${ }^{11}$

Cheng et al. performed an extensive aggregated-level data meta-analysis for associations of both former and current smoking with VTE and showed statistically significant associations (relative risks of 1.26 [95\% CI, 1.16-1.37] for current and 1.07 [1.04-1.11] for former smoking in the cohort studies). ${ }^{12}$ In our VTE subtype analyses, the association of smoking with provoked VTE (fully adjusted HR=1.36 and 1.11 for current and former smoking, respectively) and the association of former smoking with unprovoked VTE were similar to the observation of Cheng et al, but we observed no association between current smoking and unprovoked VTE that they observed (relative risk of 1.28 [1.16-1.42]). However, besides adjustment for different sets of covariates across the included studies and inclusion of nonvalidated VTE events, the number of studies contributing to each analysis differed across VTE subtypes in the meta-analysis by Cheng et al, which complicates interpretations. The stronger association of smoking with provoked VTE in our meta-analysis could be explained by the well-known association of smoking with cancer, and/or increased risk of hospitalization for respiratory illnesses, myocardial infarction and stroke causing immobilization. This speculation is supported by the results of the Iowa Women's Health Study and the Troms $\varnothing$ study. ${ }^{26,27}$ The latter study showed that the apparent association between smoking and provoked VTE disappeared in cause-specific analyses where individuals were censored at the occurrence of first cancer or myocardial infarction. ${ }^{27}$

In the current meta-analysis, continuous blood pressure measures, in particular systolic blood pressure, showed an inverse association with VTE risk. One previous study, which is included in our meta-analysis, also found an inverse association of blood pressure with VTE. ${ }^{28}$ Exclusion of this study did not alter the associations (data not shown). It is possible that the inverse association is due to the competing risk of comorbid conditions such as atrial fibrillation, which is strongly associated with high blood pressure, with subsequent anticoagulant drug use being protective against VTE. Moreover, some antithrombotic effects have been described for antihypertensive drugs such as the angiotensin converting enzyme inhibitors. ${ }^{29}$

Our meta-analysis has some limitations. Although care was taken to harmonize the definitions across studies, some differences remained such as differences in assays and blood pressure measurement devices. Definitions of provoked VTE varied to some extent across studies as listed in the online-only Data Supplement pages 3-8. However, there was little 
evidence for heterogeneity across studies. Some experts consider a one-stage meta-analysis of individual participant data as the best method of evidence synthesis. However, in the setting of the same pre-specified definitions and cutoff values across all studies, the twostage method of individual participant data provide the same estimates as the one-stage method. ${ }^{30}$ Information on the use of anticoagulant and antithrombotic drugs at baseline and during follow-up was not available in most studies. Results of the current meta-analysis may not be generalizable to non-Caucasian populations given that in most studies primarily Caucasian individuals were enrolled. In general a long-term follow-up without repeated measures may introduce regression dilution bias, however, in our analyses it seems unlikely given that the results were similar in the sensitivity analyses of limiting follow-up to the first 5 years. Finally, the results of particularly the continuous analyses should be interpreted in light of clinical significance as due to high statistical power very small and clinically irrelevant association were sometimes statistically significant (e.g., normal range of lipid measures and unprovoked VTE or normal range of glucose levels and overall/subtypes of VTE). Despite these limitations, this individual participant data meta-analysis provides conclusive evidence on the association of CVD risk factors with VTE.

In conclusion, in this individual-level data meta-analysis of prospective studies with measured CVD risk factors in nearly 250,000 participants and nearly 5,000 validated VTE events, the modifiable traditional CVD risk factors were not associated with increased risk of VTE, with the exception of the association of cigarette smoking with provoked VTE. Our findings suggest that previously reported positive associations of traditional CVD risk factors with VTE are likely due to not accounting for confounding factors.

\section{Supplementary Material}

Refer to Web version on PubMed Central for supplementary material.

\section{Acknowledgments}

Individual studies were supported by variety of sources for enrollment and data collection including laboratory measurements, and follow-up (Online-only Data Supplement pages 3-8). Current meta-analysis was sponsored by a small grant $(<\$ 10,000)$ provided by the Sint Antonius Hospital's research fund.

Role of the Funding Source: The sponsors had no role in the study design, analysis, and data interpretation or writing of the manuscript. BKM had full access to the output and estimates of the studies and performed the metaanalysis of the estimates; individual-level data were accessible by the representing co-author of the individual studies. All authors had final responsibility for the decision to submit for publications.

\section{References}

1. Beckman MG, Hooper WC, Critchley SE, Ortel TL. Venous thromboembolism: a public health concern. Am J Prev Med. 2010; 38:S495-501. [PubMed: 20331949]

2. Cohen AT, Agnelli G, Anderson FA, Arcelus JI, Bergqvist D, Brecht JG, Greer IA, Heit JA, Hutchinson JL, Kakkar AK, Mottier D, Oger E, Samama MM, Spannagl M, Europe VTEIAGi. Venous thromboembolism (VTE) in Europe. The number of VTE events and associated morbidity and mortality. Thromb Haemost. 2007; 98:756-64. [PubMed: 17938798]

3. Rosendaal FR. Risk factors for venous thrombotic disease. Thromb Haemost. 1999; 82:610-9. [PubMed: 10605758]

4. White RH. The epidemiology of venous thromboembolism. Circulation. 2003; 107:I4-8. [PubMed: 12814979] 
5. Goff DC Jr, Lloyd-Jones DM, Bennett G, Coady S, D'Agostino RB Sr, Gibbons R, Greenland P, Lackland DT, Levy D, O'Donnell CJ, Robinson J, Schwartz JS, Shero ST, Smith SC Jr, Sorlie P, Stone NJ, Wilson PW. 2013 ACC/AHA Guideline on the Assessment of Cardiovascular Risk: A Report of the American College of Cardiology/American Heart Association Task Force on Practice Guidelines. Circulation. 2013; 129:S49-73. [PubMed: 24222018]

6. Prandoni P. Venous thromboembolism and atherosclerosis: is there a link? J Thromb Haemost. 2007; 5(Suppl 1):270-5. [PubMed: 17635736]

7. Holst AG, Jensen G, Prescott E. Risk factors for venous thromboembolism: results from the Copenhagen City Heart Study. Circulation. 2010; 121:1896-903. [PubMed: 20404252]

8. Ageno W, Becattini C, Brighton T, Selby R, Kamphuisen PW. Cardiovascular risk factors and venous thromboembolism: a meta-analysis. Circulation. 2008; 117:93-102. [PubMed: 18086925]

9. Gariani K, Mavrakanas T, Combescure C, Perrier A, Marti C. Is diabetes mellitus a risk factor for venous thromboembolism? A systematic review and meta-analysis of case-control and cohort studies. Eur J Intern Med. 2016; 28:52-8. [PubMed: 26507303]

10. Bai J, Ding X, Du X, Zhao X, Wang Z, Ma Z. Diabetes is associated with increased risk of venous thromboembolism: a systematic review and meta-analysis. Thromb Res. 2015; 135:90-5. [PubMed: 25434631]

11. Bell EJ, Folsom AR, Lutsey PL, Selvin E, Zakai NA, Cushman M, Alonso A. Diabetes mellitus and venous thromboembolism: A systematic review and meta-analysis. Diabetes Res Clin Pract. 2016; 111:10-8. [PubMed: 26612139]

12. Cheng YJ, Liu ZH, Yao FJ, Zeng WT, Zheng DD, Dong YG, Wu SH. Current and former smoking and risk for venous thromboembolism: a systematic review and meta-analysis. PLoS Med. 2013; 10:e1001515. [PubMed: 24068896]

13. Braekkan SK, Hald EM, Mathiesen EB, Njolstad I, Wilsgaard T, Rosendaal FR, Hansen JB. Competing risk of atherosclerotic risk factors for arterial and venous thrombosis in a general population: the Tromso study. Arterioscler Thromb Vasc Biol. 2012; 32:487-91. [PubMed: 22075253]

14. Mahmoodi BK, Gansevoort RT, Veeger NJ, Matthews AG, Navis G, Hillege HL, van der Meer J. Microalbuminuria and risk of venous thromboembolism. JAMA. 2009; 301:1790-7. [PubMed: 19417196]

15. Tsai AW, Cushman M, Rosamond WD, Heckbert SR, Polak JF, Folsom AR. Cardiovascular risk factors and venous thromboembolism incidence: the longitudinal investigation of thromboembolism etiology. Arch Intern Med. 2002; 162:1182-9. [PubMed: 12020191]

16. Wattanakit K, Lutsey PL, Bell EJ, Gornik H, Cushman M, Heckbert SR, Rosamond WD, Folsom AR. Association between cardiovascular disease risk factors and occurrence of venous thromboembolism. A time-dependent analysis. Thromb Haemost. 2012; 108:508-15. [PubMed: 22782466]

17. Fitzgerald KC, Chiuve SE, Buring JE, Ridker PM, Glynn RJ. Comparison of associations of adherence to a Dietary Approaches to Stop Hypertension (DASH)-style diet with risks of cardiovascular disease and venous thromboembolism. J Thromb Haemost. 2012; 10:189-98. [PubMed: 22151600]

18. Glynn RJ, Danielson E, Fonseca FA, Genest J, Gotto AM Jr, Kastelein JJ, Koenig W, Libby P, Lorenzatti AJ, MacFadyen JG, Nordestgaard BG, Shepherd J, Willerson JT, Ridker PM. A randomized trial of rosuvastatin in the prevention of venous thromboembolism. N Engl J Med. 2009; 360:1851-61. [PubMed: 19329822]

19. Ray JG, Lonn E, Yi Q, Rathe A, Sheridan P, Kearon C, investigators H- Yusuf S, Arnold MJ, McQueen MJ, Pogue J, Probstfield J, Fodor G, Held C, Micks M, Genest J Jr. Venous thromboembolism in association with features of the metabolic syndrome. QJM. 2007; 100:67984. [PubMed: 17846056]

20. Rosengren A, Freden M, Hansson PO, Wilhelmsen L, Wedel H, Eriksson H. Psychosocial factors and venous thromboembolism: a long-term follow-up study of Swedish men. J Thromb Haemost. 2008; 6:558-64. [PubMed: 18045241]

21. Manson JE, Chlebowski RT, Stefanick ML, Aragaki AK, Rossouw JE, Prentice RL, Anderson G, Howard BV, Thomson CA, LaCroix AZ, Wactawski-Wende J, Jackson RD, Limacher M, Margolis 
KL, Wassertheil-Smoller S, Beresford SA, Cauley JA, Eaton CB, Gass M, Hsia J, Johnson KC, Kooperberg C, Kuller LH, Lewis CE, Liu S, Martin LW, Ockene JK, O'Sullivan MJ, Powell LH, Simon MS, Van Horn L, Vitolins MZ, Wallace RB. Menopausal hormone therapy and health outcomes during the intervention and extended poststopping phases of the Women's Health Initiative randomized trials. JAMA. 2013; 310:1353-68. [PubMed: 24084921]

22. Naess IA, Christiansen SC, Romundstad P, Cannegieter SC, Rosendaal FR, Hammerstrom J. Incidence and mortality of venous thrombosis: a population-based study. J Thromb Haemost. 2007; 5:692-9. [PubMed: 17367492]

23. Severinsen MT, Kristensen SR, Johnsen SP, Dethlefsen C, Tjonneland A, Overvad K. Anthropometry, body fat, and venous thromboembolism: a Danish follow-up study. Circulation. 2009; 120:1850-7. [PubMed: 19858417]

24. Zakai NA, McClure LA, Judd SE, Safford MM, Folsom AR, Lutsey PL, Cushman M. Racial and Regional Differences in Venous Thromboembolism in the United States in Three Cohorts. Circulation. 2014; 129:1502-9. [PubMed: 24508826]

25. Puurunen MK, Enserro D, Xanthakis V, Larson MG, Benjamin EJ, Tofler GH, Wollert KC, O'Donnell CJ, Vasan RS. Biomarkers for the prediction of venous thromboembolism in the community. Thromb Res. 2016; 145:34-39. [PubMed: 27447083]

26. Lutsey PL, Virnig BA, Durham SB, Steffen LM, Hirsch AT, Jacobs DR Jr, Folsom AR. Correlates and consequences of venous thromboembolism: The Iowa Women's Health Study. Am J Public Health. 2010; 100:1506-13. [PubMed: 19910349]

27. Enga KF, Braekkan SK, Hansen-Krone IJ, le Cessie S, Rosendaal FR, Hansen JB. Cigarette smoking and the risk of venous thromboembolism: the Tromso Study. J Thromb Haemost. 2012; 10:2068-74. [PubMed: 22882779]

28. Quist-Paulsen P, Naess IA, Cannegieter SC, Romundstad PR, Christiansen SC, Rosendaal FR, Hammerstrom J. Arterial cardiovascular risk factors and venous thrombosis: results from a population-based, prospective study (the HUNT 2). Haematologica. 2010; 95:119-25. [PubMed: 19713225]

29. Wojewodzka-Zelezniakowicz M, Chabielska E, Mogielnicki A, Kramkowski K, Karp A, Opadczuk A, Domaniewski T, Malinowska-Zaprzalka M, Buczko W. Antithrombotic effect of tissue and plasma type angiotensin converting enzyme inhibitors in experimental thrombosis in rats. J Physiol Pharmacol. 2006; 57:231-45. [PubMed: 16845228]

30. Stewart GB, Altman DG, Askie LM, Duley L, Simmonds MC, Stewart LA. Statistical analysis of individual participant data meta-analyses: a comparison of methods and recommendations for practice. PLoS ONE. 2012; 7:e46042. [PubMed: 23056232] 


\section{Clinical Perspective}

\section{What Is New?}

- $\quad$ This first individual level-data meta-analyses of prospective studies demonstrates that traditional cardiovascular risk factors are not independent risk factors for venous thromboembolism (VTE).

- $\quad$ Cigarette smoking in particular current smoking was associated with mildly elevated risk of provoked VTE, which may be mediated through comorbid conditions such as cancer.

- Higher systolic blood pressure showed inverse association with VTE risk, which may be due to competing risk of comorbid conditions such as atrial fibrillation, which is strongly associated with high blood pressure, with subsequent anticoagulant drug use being protective against VTE.

\section{What Are the Clinical Implications?}

- $\quad$ Traditional risk factors for venous and arterial disease differ, supporting different pathogenesis of these thrombotic disorders.

- $\quad$ Traditional CVD risk factors should not be used to assess risk of (first) VTE. 
Flowchart

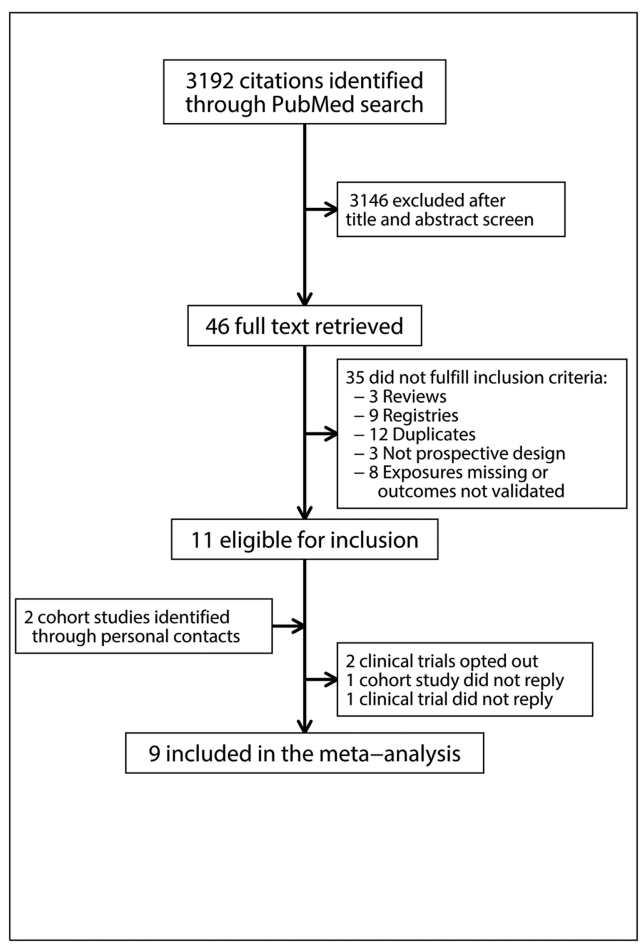

Figure 1. Flow diagram for selection of studies 


\begin{tabular}{|c|c|c|c|c|}
\hline Variables & Models & N VTE/ Part. & Hazard Ratio & HR (95\% Cl) \\
\hline \multirow{3}{*}{ Hypertension } & Model 1 & $4900 / 244198$ & $\rightarrow$ & $1.59(1.30-1.94)$ \\
\hline & Model 2 & $4870 / 243010$ & & $0.98(0.89-1.07)$ \\
\hline & Model 3 & $4639 / 233383$ & & $0.98(0.91-1.05)$ \\
\hline \multirow{3}{*}{ Hyperlipidemia } & Model 1 & $3957 / 184205$ & $\rightarrow$ & $1.28(1.00-1.65)$ \\
\hline & Model 2 & $3934 / 183163$ & & $0.97(0.88-1.07)$ \\
\hline & Model 3 & $3854 / 177476$ & & $0.96(0.88-1.05)$ \\
\hline \multirow{3}{*}{ Diabetes } & Model 1 & $4882 / 243366$ & $\longrightarrow$ & $1.50(1.26-1.80)$ \\
\hline & Model 2 & $4850 / 241957$ & & $1.01(0.89-1.15)$ \\
\hline & Model 3 & $4639 / 233383$ & & $1.00(0.89-1.13)$ \\
\hline \multirow{3}{*}{ Current smoking } & Model 1 & $4861 / 240104$ & - & $0.87(0.72-1.05)$ \\
\hline & Model 2 & $4830 / 238747$ & $\rightarrow$ & $1.19(1.08-1.32)$ \\
\hline & Model 3 & $4639 / 233383$ & $\rightarrow$ & $1.21(1.07-1.37)$ \\
\hline \multirow{3}{*}{ Former smoking } & Model 1 & $4087 / 169346$ & -6 & $1.13(1.03-1.25)$ \\
\hline & Model 2 & $4070 / 168749$ & & $0.99(0.92-1.05)$ \\
\hline & Model 3 & $3881 / 163848$ & 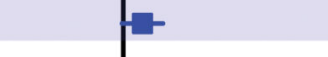 & $1.07(0.99-1.15)$ \\
\hline & & $\Gamma$ & & \\
\hline & & 0.5 & 1 & \\
\hline
\end{tabular}

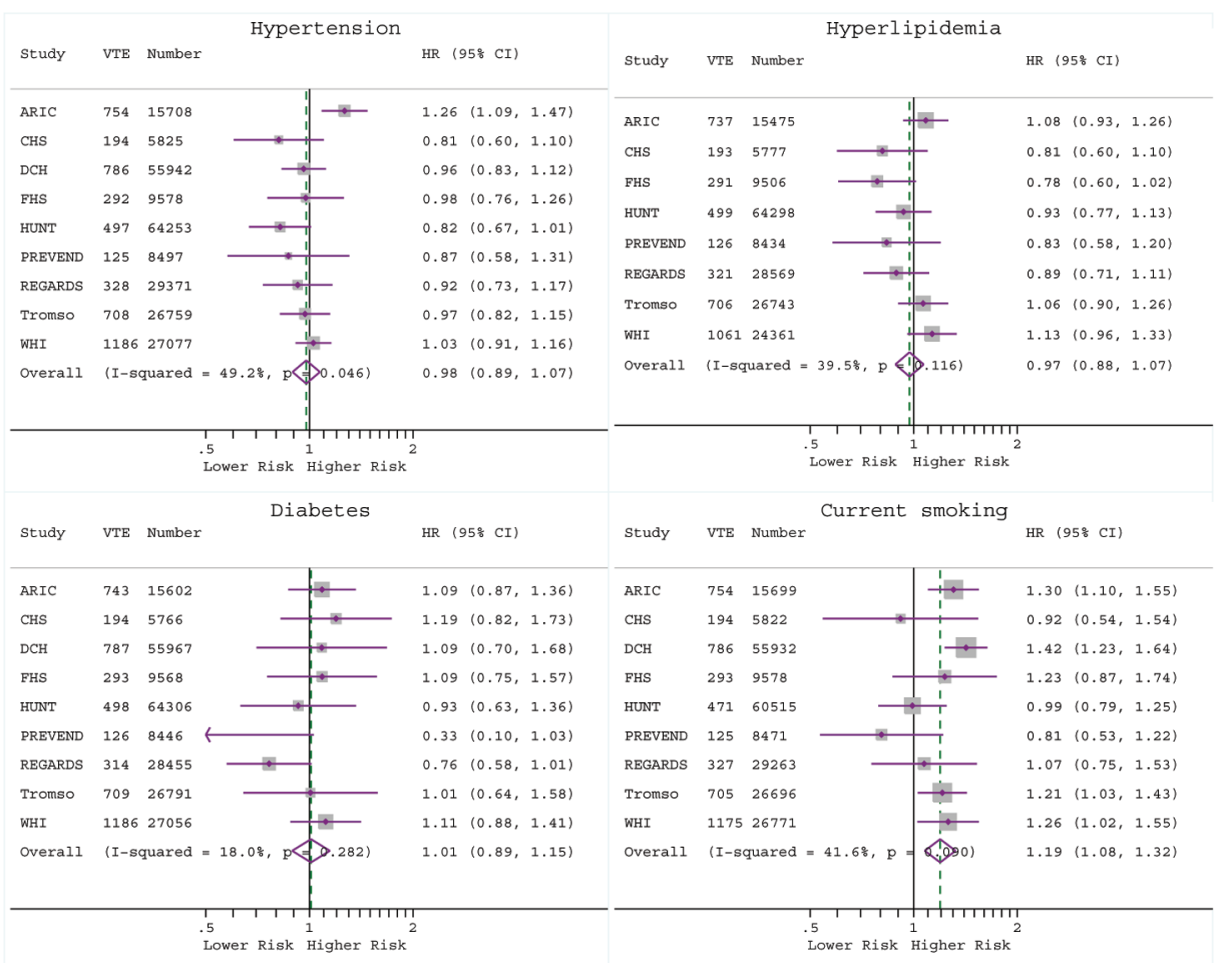

Figure 2. Pooled and study-specific hazard ratios of overall VTE

The top panel shows the pooled estimates from crude models (Model 1); age, sex and BMI adjusted models (Model 2); and fully adjusted models (Model 3). The fully adjusted model included age, sex, race, BMI (continuous), history of CVD, history of VTE, hypertension, hypercholesterolemia, diabetes, former and current smoking. The bottom panel shows study- 
specific hazard ratios of overall VTE for hypertension, hypercholesterolemia, diabetes, and current smoking adjusted for age, sex and BMI. VTE denotes venous thromboembolism; and Part., participants. 

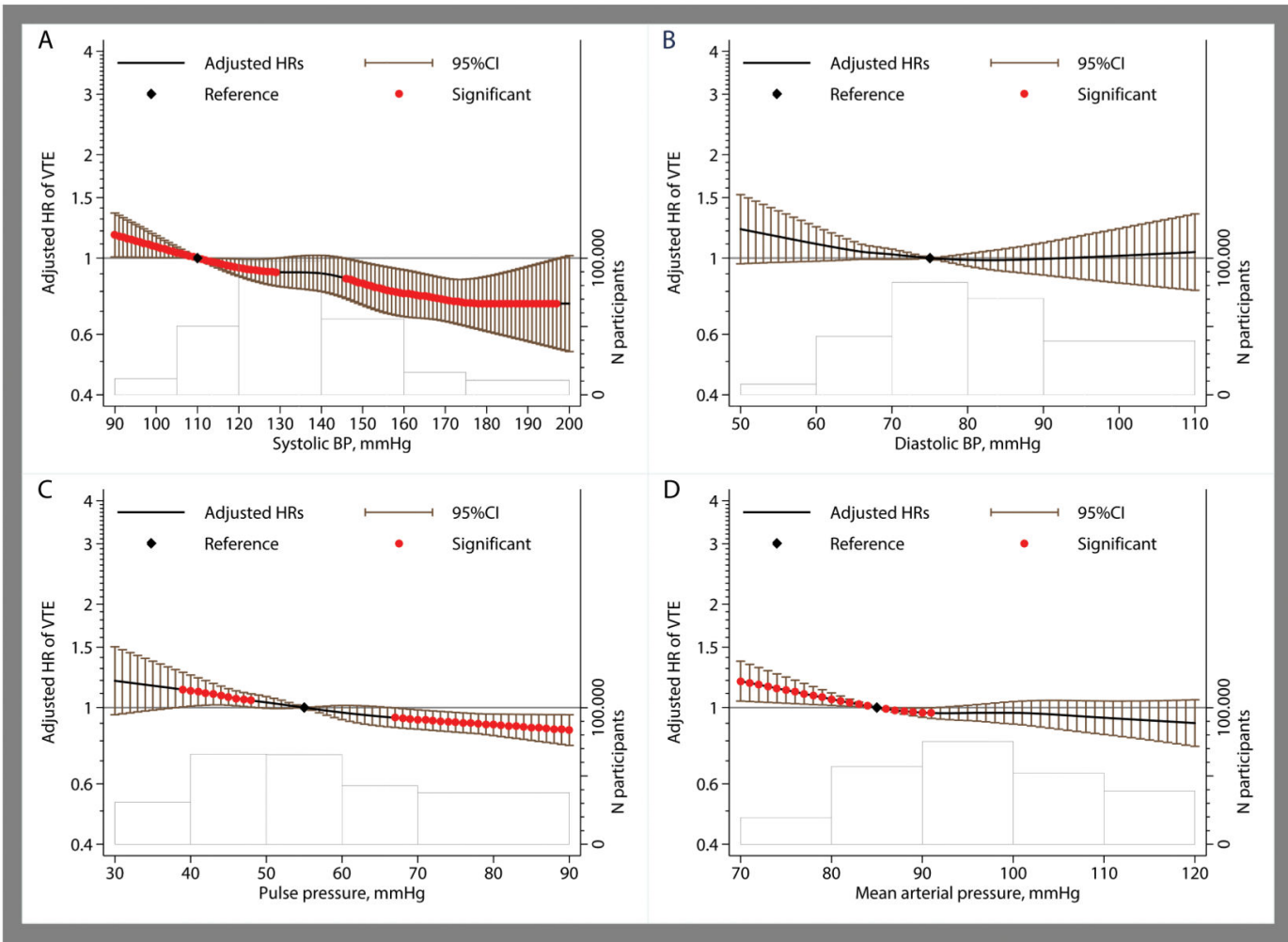

Figure 3. Pooled hazard ratios of overall VTE according to blood pressure measurements for systolic pressure (panel A), diastolic pressure (panel B), pulse pressure (Panel C) and mean arterial pressure (Panel D)

Estimates are adjusted for age, sex, BMI (continuous), history of cardiovascular disease and antihypertensive medication use. The black line and the error-bars depict hazard rations and $95 \%$ confidence interval with the red dots indicating statistical significance $(\mathrm{P}<0.05)$ and the black diamond the reference value. The heights of the bars shown with the gray lines at the bottom of each graph depict the number of participants at each spline section and the widths of these bars correspond to the splines range. 


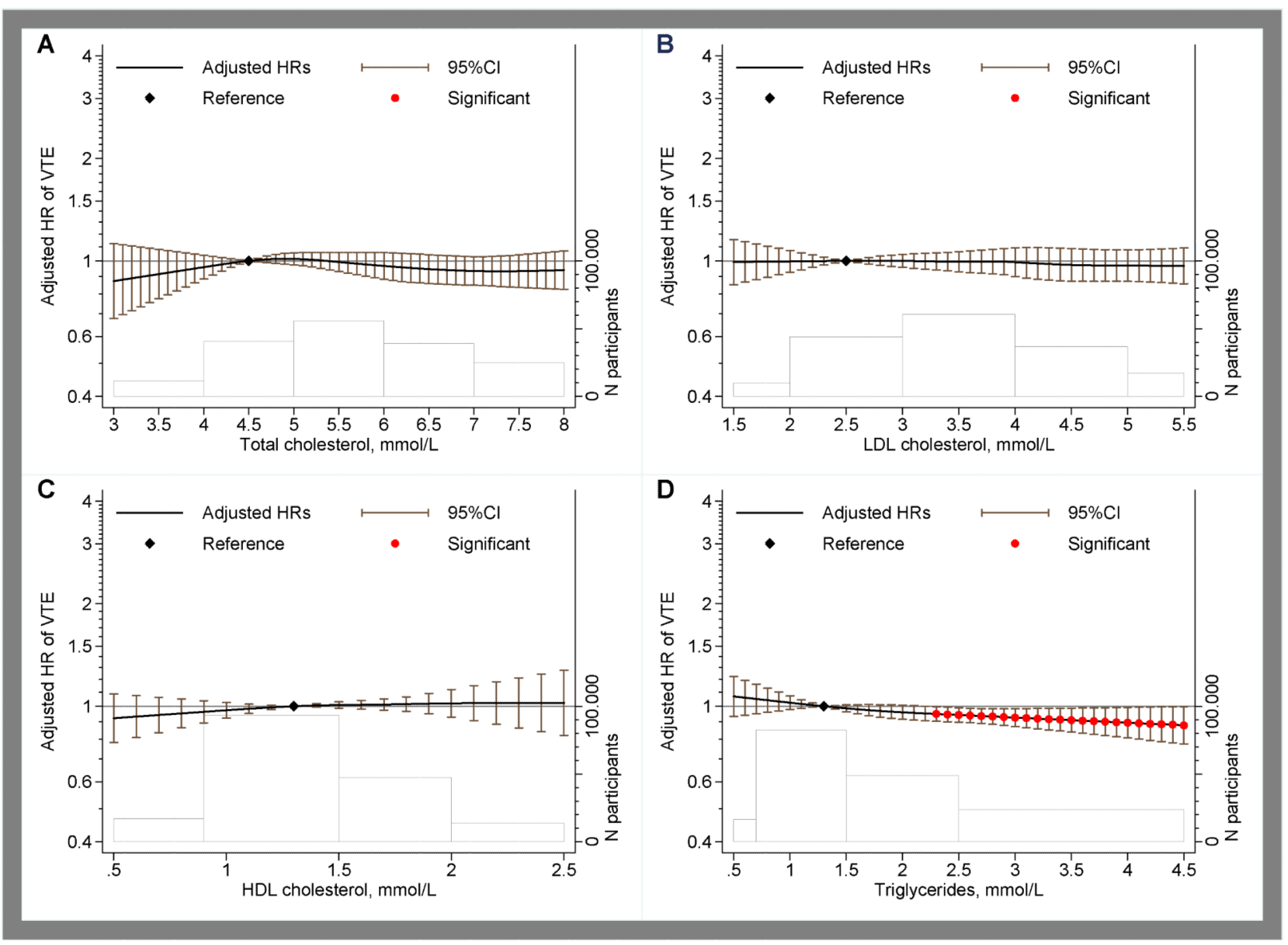

Figure 4. Pooled hazard ratios of overall VTE according to lipid measurements for total cholesterol (panel A), low-density lipoproteins (panel B), high-density lipoproteins (Panel C) and triglycerides (Panel D) levels

Estimates are adjusted for age, sex, BMI (continuous), history of cardiovascular disease and lipid-lowering medication use. The black line and the error-bars depict hazard rations and $95 \%$ confidence interval with the red dots indicating statistical significance $(\mathrm{P}<0.05)$ and the black diamond the reference value. The heights of the bars shown with the gray lines at the bottom of each graph depict the number of participants at each spline section and the widths of these bars correspond to the splines range. 


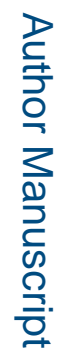

要

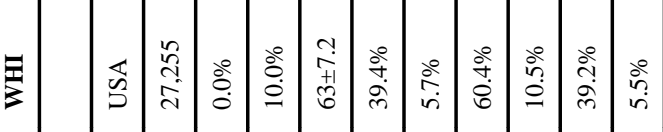

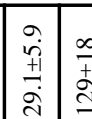

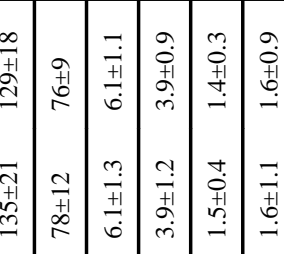

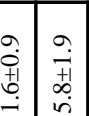

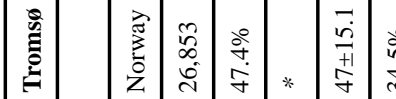

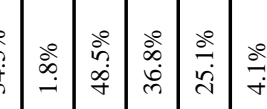

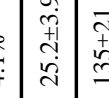

㤏

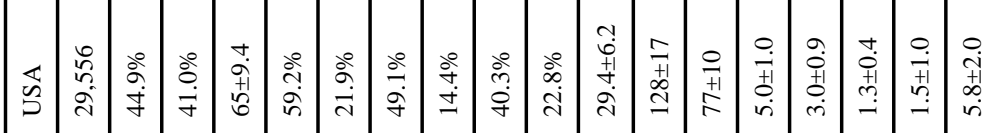

育

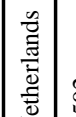

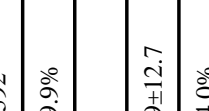

$\therefore$

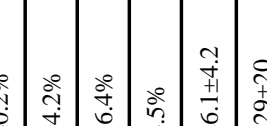

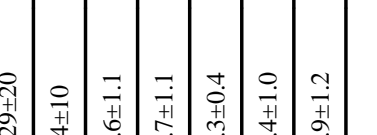

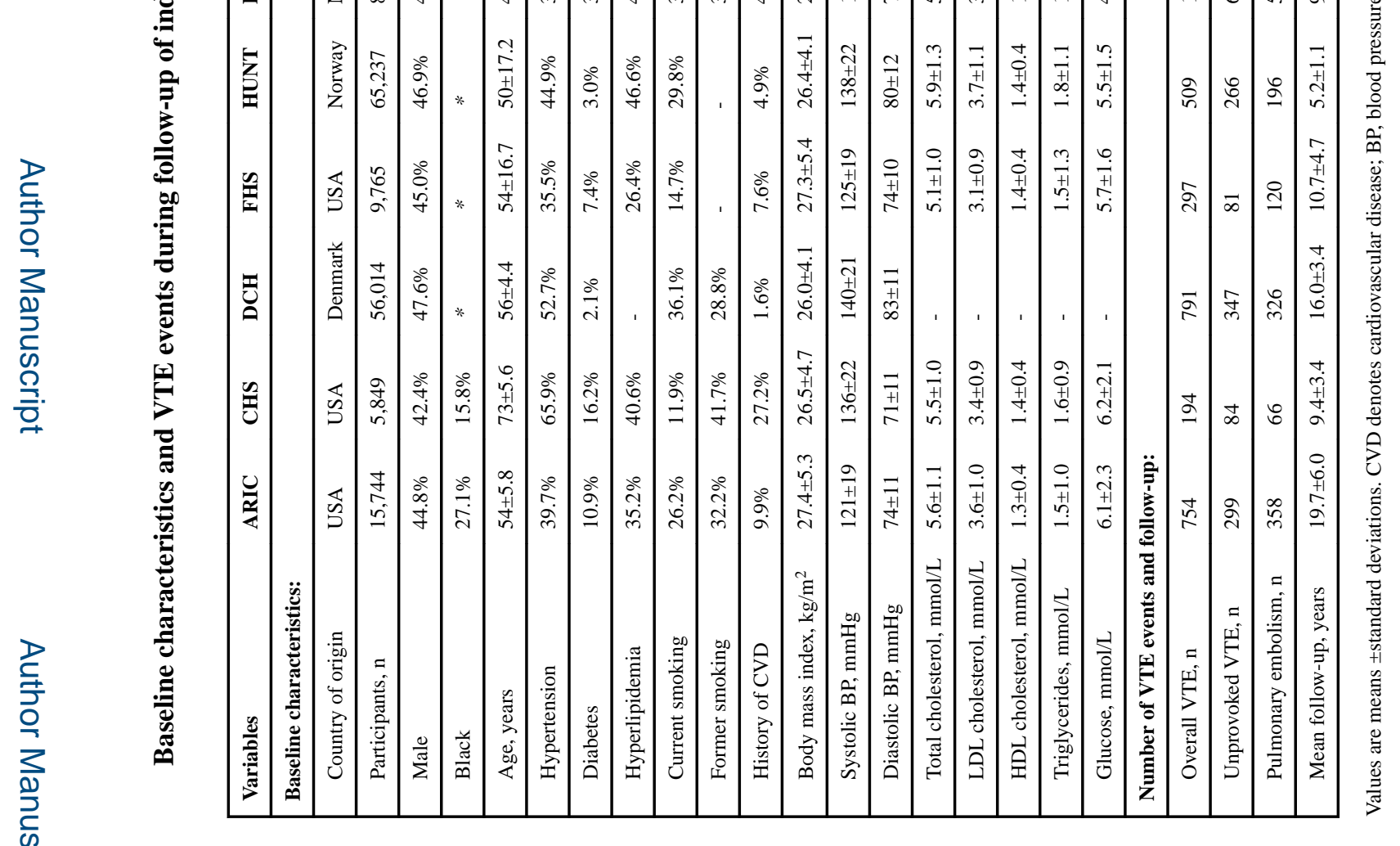

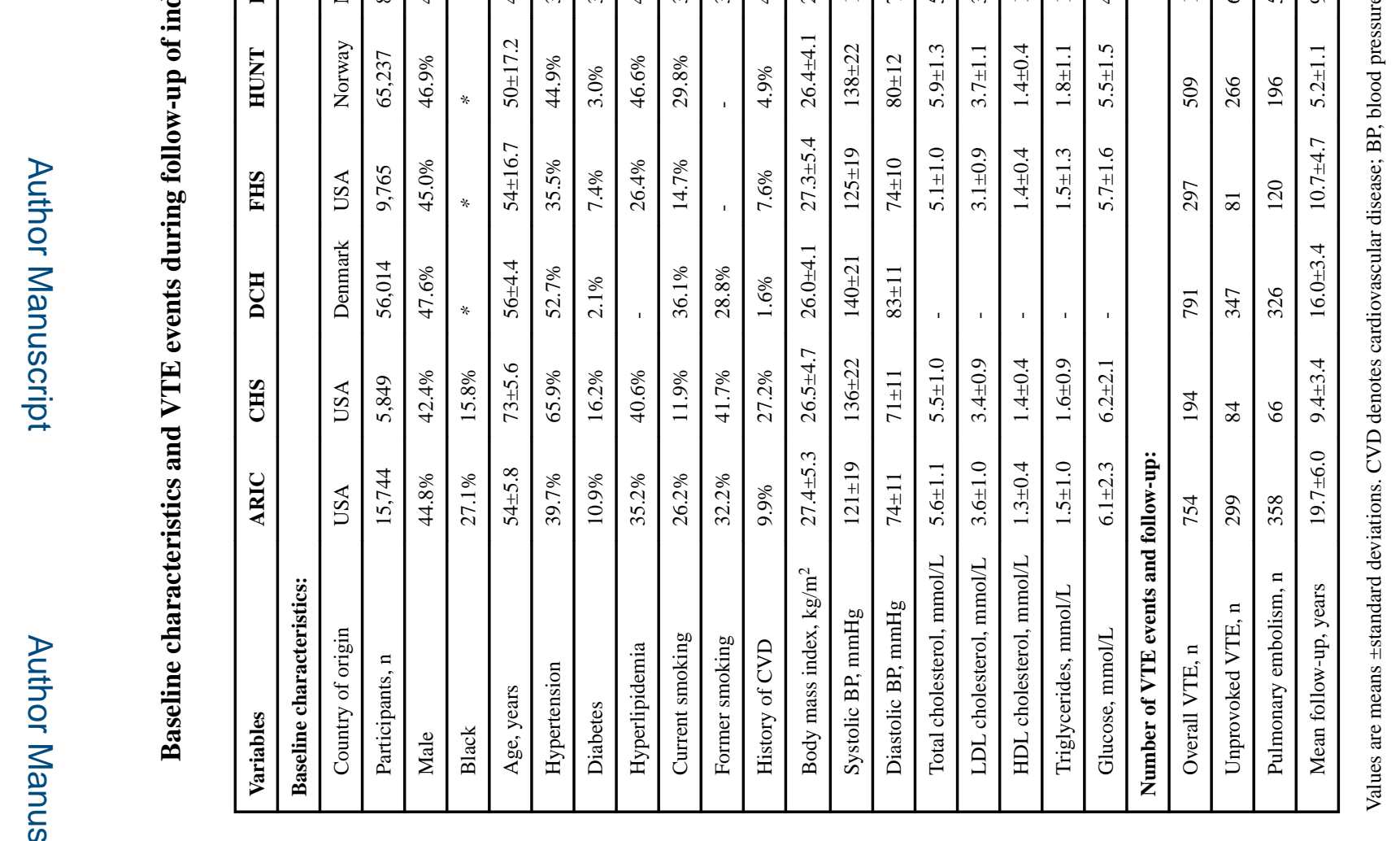

로을

Circulation. Author manuscript; available in PMC 2018 January 03. 
